\title{
Estudio de la calidad del aceite de oliva virgen de la Denominación de Origen «Les Garrigues» (Lleida) durante la campaña 1995/96
}

\author{
Por Ma José Motilva*, Ismael Jaria, Ismael Bellart y Mª Paz Romero
}

Departamento de Tecnología de Alimentos, Escuela Técnica Superior de Ingeniería Agraria, Universidad de Lleida. Centro UdL-IRTA. Avda. Alcalde Rovira Roure, 177. 25198 Lleida, España.

\section{RESUMEN}

Estudio de la calidad del aceite de oliva virgen de la Denominación de Origen "Les Garrigues» (Lleida) durante la campaña 1995/96.

La Denominación de Origen "Les Garrigues" se sitúa en el extremo meridional de la provincia de Lleida. La gran mayoría del aceite producido en esta D.O. corresponde a la categoria de virgen extra y posee unas características organolépticas muy peculiares, derivadas del fruto de origen, la variedad "Arbequina" y de su cuidada elaboración. La aplicación en la zona de modernas técnicas de cultivo del olivo y las nuevas tecnologías de extracción del aceite, hacen necesario un estudio de su composición en campañas sucesivas, con el fin de determinar si la calidad se pudiera ver afectada. El presente trabajo se centra en la caracterización de los aceites producidos en la D.O. "Les Garrigues" durante la campaña 1995/96.

Los aceites obtenidos presentan un grado de acidez medio de $0.17 \%$, índice de peróxidos de $6.9 \mathrm{meq} / \mathrm{kg}$ de aceite y un $\mathrm{K}_{270}$ de 0.21 , valores todos ellos dentro de los criterios de calidad que establece la Reglamentación de la D.O. El contenido medio de polifenoles totales en el aceite es de 152 ppm, observándose una gran variabilidad entre las almazaras muestreadas. La concentración de clorofilas va de $15 \mathrm{mg} / \mathrm{kg}$ de aceite, al inicio de campaña, a $6 \mathrm{mg} / \mathrm{kg}$ al final de la misma. La concentración de carotenoides se reduce paralelamente, pasando de 12.6 a $4.6 \mathrm{mg} / \mathrm{kg}$ de aceite, al final de campaña. El color, en base a la escala visual ABT y a las coordenadas CIELAB, se mantiene dentro de los tonos del amarillo y no presenta diferencias importantes entre aceites de principio y final de campaña.

PALABRAS-CLAVE: Aceite de oliva - Calidad - Color Pigmento - Polifenol - Variedad arbequina.

\section{SUMMARY}

Quality of virgin olive oil from territorial quality label "Les Garrigues" D.O. (Lleida. Spain) along the 1995/96 harvest.

The Garrigues territorial quality label (Les Garrigues D.O.) is located in the southern part of Lleida (Catalonian. Spain). The most oil produced in this D.O. corresponds to extra virgin classification and it has a distinctive sensorial quality, as a consecuence of olive cultivar ("Arbequina" cv) and the careful elaboration of oil. The new cultural technologies applied to the olive tree cultivar and the new technologies of olive oil extraction have made necessary the study of oil composition and quality during consecutive harvests, to know if the quality could be afected. The objective of this work is the characterization of virgin olive oils (67 samples from 13 olive mills) during the 1995/96 harvest.
Analytical controls show that the mean acidity index is $0.17 \%$ in oleic acid, the mean peroxide value is 6.9 meq oxygene active $/ \mathrm{kg}$ of oil and the mean $\mathrm{K}_{270}$ of 0.21 ; these quality parameters fulfill the policy values of "Les Garrigues" D.O. The mean polyphenol content is $152 \mathrm{ppm}$ expressed as cafeic acid, with a great variability among olive mills. As harvesting time progresses, the concentration of pigments in olive oil decreases. The concentration of chlorophyl and that of carotenoid pigments at the begin of the harvest is 15 and $12.6 \mathrm{mg} / \mathrm{kg}$ of oil respectively and at the end of the period they are 6 and $4.6 \mathrm{mg} / \mathrm{kg}$ of oil respectively. The oil color, expressed as visual $A B T$ index and the chromatic ordinates CIELAB, lies within the yellow zone and does not vary along the harvest season.

KEY-WORDS: Arbequina variety - Colour - Olive oil Pigment - Polyphenol - Quality.

\section{INTRODUCCIÓN}

El aceite de oliva, en el ámbito catalán, se puede dividir en tres zonas geográficas que producen aceites de características propias y bien diferenciadas. Dos de estas zonas están amparadas por las Denominaciones de Origen «Les Garrigues» (inicialmente «Borjas Blancas») y "Siurana», en las provincias de Lleida y Tarragona respectivamente, y la tercera corresponde a la zona del Baix Ebre-Montsia en Tarragona, que se caracteriza por una mayor producción de aceite de oliva, pero de menor calidad que las D.O. (Garcia Guillamet, 1995).

La Denominación de Origen «Les Garrigues» se sitúa en el extremo meridional de la provincia de Lleida y abarca 42 términos municipales de la comarca de Les Garrigues, parte del Urgell y del Segria. El área geográfica delimitada por la zona de producción de la D.O. abarca una extensión de terreno de características topográficas y climáticas similares, de manera que el aceite de oliva producido es prácticamente homogéneo en cuanto a la influencia del medio físico, tal como pretende la reglamentación de las denominaciones de origen. En la figura 1 se muestra el mapa de la D.O. "Les Garrigues", en donde se han señalado las cooperativas almazareras existentes. 


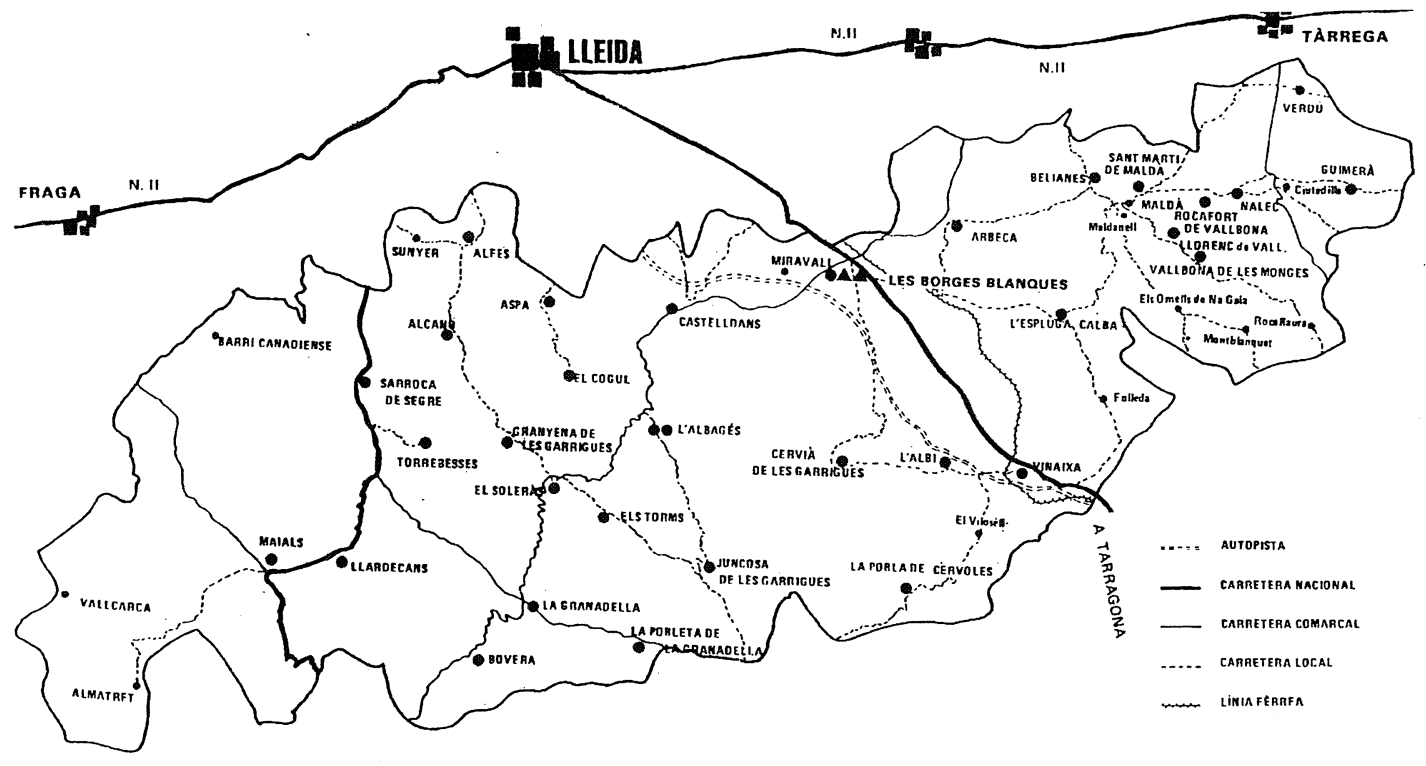

Figura 1

Mapa de la zona de producción de aceite de oliva virgen de la D.O. «Les Garrigues» (Lleida)

El paisaje más característico de esta zona se compone por un sistema de pequeños altiplanos, que se alternan con valles y torrentes secos de laderas abancaladas, característico de Les Garrigues Altes y de la Segarra Baixa, subcomarca del Urgell. Por el contrario, en la zona de Les Garrigues Baixes y del Segria, predominan las pendientes suaves, además de las aportaciones de agua de riego del Canal de Urgell, en la zona norte. El clima es de tipo mediterráneo continental, caracterizado por veranos calurosos y secos, con máximas que superan los $40^{\circ} \mathrm{C}$, e inviernos fríos y largos. Esta acusada diferencia térmica distingue esta zona de las otras zonas catalanas productoras de aceite de oliva, con un clima más atemperado por la proximidad del Mediterráneo. Las precipitaciones muy escasas e irregulares, varían desde los $400 \mathrm{~mm}$ en el norte, hasta los más de 550 $\mathrm{mm}$ en las zonas más altas (Pujades, 1984).

La variedad dominante en la D.O. "Les Garrigues» es la «Arbequina», que constituye el $90 \%$ del olivar y prácticamente sólo se utiliza para la elaboración de aceite. La mayoría de las plantaciones datan del siglo pasado y son de pequeña dimensión y baja densidad (Tous y Romero, 1993).

El aceite de oliva virgen de la D.O. «Les Garrigues" tiene unas características organolépticas muy peculiares, derivadas de la variedad del fruto de origen y de su cuidada elaboración, que permite mantener el grado de acidez del aceite por debajo de $0.5 \%$. Según el momento de recolección se distinguen dos tipos de aceite. "Frutado», de recolección más temprana, color verdoso, más cuerpo y sabor almendrado-amargo, y el denominado "dulce», de recolección más tardía, color amarillo, más fluido y sabor «dulzón».

Son numerosos los estudios que se han realizado hasta el momento, orientados al estudio de la calidad de estos aceites en campañas sucesivas
(Graell, 1992; Tous y Romero, 1994; Romero et al., 1995a; Solé, 1995a y b). Todos ellos han mostrado bajos valores de acidez, con medias que oscilan en torno al $0.18 \%$, aunque en ocasiones han alcanzado valores de $2.30 \%$, relacionados con épocas de su- perproducción de oliva y con el sistema tradicional de prensado. El índice de peróxidos, por lo general se mantiene por debajo de los máximos normativos, en torno a $6 \mathrm{meq} / \mathrm{kg}$ de aceite, aunque hay que señalar la dispersión de valores dentro de la D.O., que en ocasiones pueden llegar a $20 \mathrm{meq} / \mathrm{kg}$ de aceite, probablemente como consecuencia de las condiciones del proceso de obtención del aceite, o bien de las intensas heladas que en alguna ocasión han afectado a la zona en plena campaña de recolección (Graell et al., 1993a, 1993b; Romero et al., 1995b). El contenido de polifenoles totales de estos aceites es bajo, en torno a las 260 ppm, lo que contribuye a que su estabilidad sea también relativamente baja (inferior a 12 horas a $120^{\circ} \mathrm{C}$ por el método Rancimat) (Romero et al., 1995a).

La actual recuperación del mercado del aceite de oliva está impulsando la aplicación en la zona de modernas técnicas de cultivo del olivo de la variedad «Arbequina», con el fin de mejorar la producción y la calidad de la materia prima. Asimismo, la introducción de nuevas tecnologías de extracción del aceite, concretamente la creciente sustitución del sistema de centrifugación contínuo de tres a dos fases, hace necesario un estudio de la composición de los aceites obtenidos en campañas sucesivas, con el fin de determinar si la calidad se puede ver afectada.

Según el Consejo Regulador de la D.O. «Les Garrigues", la producción de aceite de oliva virgen en esta denominación para la campaña 1995/96 se ha situado en unas $4.700 \mathrm{tm}$, que se corresponde con la producción media de la zona que oscila entre las 4.500 y 6.000 tm de aceite virgen. A nivel climatoló- 
gico ha sido un año atípico en la zona; la sequía de los últimos años se ha agravado durante el periodo vegetativo del olivo y durante la maduración de la oliva, aunque en los meses de recolección las precipitaciones han aumentado por encima de la media de los años 1992/94. Las cálidas temperaturas de invierno y la falta de lluvias han adelantado la maduración de la oliva, de manera que varias almazaras de la D.O. ya comenzaron su actividad a finales del mes de octubre.

El objetivo del presente trabajo se centra en el estudio de la calidad de los aceites de oliva virgen de la D.O. «Les Garrigues» durante la campaña 1995/96. El estudio se plantea desde el punto de vista de parámetros clásicos de calidad: grado de acidez, índice de peróxidos y absorción en el U.V., y nuevos criterios de calidad tales como contenido de polifenoles totales, pigmentos (clorofilas y carotenos) y color. En todos los parámetros analizados, se estudiará la diferencia entre las distintas almazaras muestreadas, con el fin de determinar la implicación de factores geográficos (altitud), climáticos (temperatura y pluviometría) y/o tecnológicos (sistema de extracción). Por otro lado, se analizarán los resultados de cada parámetro a lo largo de la campaña, con el objetivo de establecer si hay diferencias entre aceites de principio y final de la misma.

\section{PARTE EXPERIMENTAL}

\subsection{Muestras}

El estudio se ha realizado sobre un total de 67 muestras, siendo la gran mayoría de ellas aceites vírgenes extra, procedentes de 13 almazaras comprendidas en la Denominación de Origen «Les Garrigues» (Lleida), durante el transcurso de la campaña 1995/96. Las almazaras-cooperativas adheridas a la Denominación se han seleccionado en base a su localización geográfica, de manera que representaran los distintos puntos de la comarca de les Garrigues; y en base a su capacidad de producción, seleccionando las de mayor producción y combinando almazaras que trabajan con sistemas de extracción continuos de dos y tres fases. En las tablas de resultados las cooperativas muestreadas se numeran del 1 al 13. Las almazaras $1,7,8,10,11$ y 12 trabajan con el sistema de extracción continua de tres fases y las almazaras 2, 3, 4, 5, 6, 9 y 13 con el sistema de extracción continua de dos fases.

La toma de muestras se ha llevado a cabo a lo largo de la campaña, en el periodo comprendido entre el 29 de octubre de 1995 y el 12 de enero de 1996, de manera que se han realizado entre 4 y 6 muestreos de cada cooperativa. Las muestras de aceite se han tomado de los depósitos de almacena- miento, con la ayuda de un recipiente que se introduce desde el fondo hacia la superficie, asegurando así la homogeneidad del muestreo. Los aceites son introducidos en frascos de vidrio topacio y, una vez en el laboratorio, se filtran y se mantienen en oscuridad hasta el momento de su análisis.

En las tablas de resultados, las semanas en las que se han realizado las tomas de muestras de aceite se han numerado del 1 al 11 , que corresponden a las siguientes fechas: 1 (29 octubre-4 noviembre de 1996), 2 (5 noviembre-11 noviembre de 1996), 3 (12 noviembre-18 noviembre de 1996), 4 (19 noviembre25 noviembre de 1996), 5 (26 noviembre-2 diciembre de 1996), 6 (3 diciembre-9 diciembre), 7 ( 10 diciembre-16 diciembre), 8 ( 17 diciembre-23 diciembre), 9 (24 diciembre-30 diciembre), 10 (31 diciembre- 6 enero) y 11 (7 enero-13 enero).

\subsection{Métodos analíticos}

\subsubsection{Parámetros de calidad}

Para la determinación de los índices generales de calidad del aceite de oliva virgen se ha seguido la metodología descrita en el Reglamento no 2568/91 de la C.E.E., del 11 de julio de 1991 (D.O.C.E. no L 248 , del 5 de septiembre de 1991) en lo relativo al grado de acidez, índice de peróxidos y prueba espectrofotométrica en el ultravioleta. Los resultados de grado de acidez se expresan en porcentaje de ácido oleico, el índice de peróxidos en miliequivalentes de oxígeno activo por kilogramo de aceite y la prueba espectrofotométrica en el ultravioleta como el coeficiente de extinción a $270 \mathrm{~nm}\left(\mathrm{~K}_{270}\right)$.

\subsubsection{Contenido de polifenoles totales}

La extracción y cuantificación de la fracción polifenólica de los aceites se han realizado en base al método descrito por Vázquez et al. (1973).

La cuantificación se realiza en base a la formación de un complejo coloreado con el reactivo de Folin-Ciocalteau (Beltran et al., 1995). Los resultados se expresan en ppm de ácido cafeico ( $\mathrm{mg}$ ácido cafeico/kg de aceite).

\subsubsection{Contenido en pigmentos}

La cuantificación global de pigmentos (carotenoides y clorofilas) se basa en la disolución de una muestra de aceite de oliva en ciclohexano y la lectura espectrofotométrica a 472 y $670 \mathrm{~nm}$, respectivamente (Mínguez et al., 1991).

La fracción carotenoide se cuantifica mediante la medida de la absorbancia a $470 \mathrm{~nm}$, que correspon- 
de a la luteina $(70 \%$ del total de los pigmentos carotenoides) y la fracción clorofílica por la absorbancia a $670 \mathrm{~nm}$ que corresponde a la feofitina, componente mayoritario de esta fracción.

La concentración de pigmentos clorofílicos y carotenoides se realiza aplicando la siguiente fórmula (Papaseit, 1986):

$$
C=\frac{E \times V_{f}}{E_{1 \%} \times p} \times 10.000
$$

\begin{tabular}{|c|c|c|}
\hline 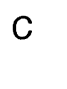 & $=$ & $\begin{array}{l}\text { concentración (mg de clorofilas o carotenos } / \mathrm{kg} \\
\text { de aceite) }\end{array}$ \\
\hline$l_{t}$ & $=$ & volumen final del extracto de pigmentos (ml) \\
\hline & $=$ & peso de la muestra de aceite (gramos) \\
\hline$E_{1 \%}$ & $=$ & $\begin{array}{l}\text { absorbancia específica de una disolución al } \\
1 \% \text { medida en una cubeta de } 1 \mathrm{~cm}\left(E_{1 \%}\right. \\
\left.\text { luteina }=2000 \text { y } E_{1 \%} \text { feofitina } a=613\right)\end{array}$ \\
\hline & $=$ & absorbancia a la $\lambda$ de medida \\
\hline
\end{tabular}

\subsubsection{Determinación del color}

\subsubsection{1. Índice ABT modificado}

Este índice está basado en la comparación visual del color de la muestra de aceite con un patrón de color de la escala ABT modificada para aceite de oliva virgen (Gutiérrez y Gutiérrez, 1986), preparada mediante mezcla de cantidades variables de soluciones tampón $1 / 15 \mathrm{M}$ de fosfato monopotásico y fosfato disódico, con azul de bromotimol al $0.04 \%$ como colorante. La nomenclatura de identificación de cada patrón se da en forma de fracción, de manera que el numerador se identifica con el tono del aceite (de verde a amarillo, a través de varios tonos intermedios) y el denominador con la luminosidad (decreciente desde el valor 1 hasta el 10). La comparación visual del aceite y del patrón se realiza utilizando tubos de dimensiones y calidad de vidrio idénticas, sobre un fondo blanco. El aceite está a una temperatura aproximada de $20^{\circ} \mathrm{C}$ y no presenta turbidez.

\subsubsection{Coordenadas instrumentales}

La determinación del color por métodos espectrofotométricos se realiza a partir de los valores triestímulo $X, Y$ y $Z$ y posterior cálculo de las ordenadas $L^{*}, a^{*}, b^{*}$ y $C^{*}$ del espacio CIELAB. La muestra de aceite, previamente filtrado y atemperado, se introduce en una cubeta de cuarzo. La lectura se realiza en un colorímetro Macbeth modelo Color-Eye 3000 , con la base informática OPTIVIEW versión 1.1, leyendo a $10^{\circ}$ con un iluminante $C$.

\subsubsection{Método estadístico}

La base informática utilizada ha sido el programa STATGRAPHICS versión 7.0. El análisis de la varianza se ha realizado con un nivel de significación del $99 \%$ para el contenido de polifenoles totales del aceite y del $95 \%$ para el resto de los parámetros analizados. Cuando las diferencias eran significativas, se ha aplicado un test de comparación de medias LSD.

En cada una de las variables estudiadas se realiza de forma independiente el análisis de la varianza entre almazaras, y por otro lado el análisis de la varianza entre las semanas de muestreo a lo largo de la campaña.

\section{RESULTADOS Y DISCUSIÓN}

Los índices de calidad de los aceites de la D.O. «Les Garrigues» de la campaña 1995/96 se sitúan dentro de los valores establecidos por su reglamentación (BOE, 13 de julio 1977), a pesar del año climático que ha propiciado una maduración muy avanzada de la oliva en la zona, de manera que la recolección se inició a comienzos del mes de octubre, cuando lo normal es al principio de noviembre. Tal y como se observa en la Tabla I, el grado de acidez se situa en un valor medio de $0.17 \%$, no existiendo diferencias significativas entre las cooperativas muestreadas, con la única excepción de la almazara 9 que presenta una acidez media $(0.22 \%)$ ligeramente superior al resto. El índice de peróxidos se mantiene en valores medios en torno a 6.9 meq $\mathrm{O}_{2}$ activo $/ \mathrm{kg}$ de aceite, con valores que oscilan entre 5.7 y 8.3 $\mathrm{meq} / \mathrm{kg}$ de aceite. En general, no se han observado diferencias destacables en ambos parámetros entre las almazaras muestreadas, posiblemente como consecuencia de la homogeneidad en las operaciones culturales, en la recolección de la oliva y en la extracción del aceite, debido al control por parte del Consejo Regulador de la D.O. Por otro lado, a lo largo del año no se han producido accidentes climáticos que pudieran haber afectado a los índices de calidad en zonas reducidas, de manera que se pudieran observar variaciones entre almazaras. Sin embargo se observa una gran variabilidad en el índice $K_{270}$, que va desde valores de 0.09 hasta 0.29 , valor que supera la Reglamentación de la D.O. para este parámetro (máximo 0.15). No obstante, esta dispersión no se ha podido atribuir a variables climatológicas o geográficas de la zona, pudiendo estar relacionada con las condiciones del proceso de extracción del aceite, de manera especial con las altas temperaturas aplicadas en algunas almazaras, que permiten aumentar el rendimiento en molturación pero pueden tener como consecuencia la aparición en el aceite de compuestos secundarios de oxidación. 
Tabla I

Índices de calidad: grado de acidez, índice de peróxidos y $\mathrm{K}_{270}$ del aceite de oliva virgen del período de recogida de muestras de las almazaras estudiadas en la D.O. «Les Garrigues», durante la campaña 1995/96 (*)

\begin{tabular}{|c|c|c|c|}
\hline Almazara & $\begin{array}{l}\left.\text { Grado de Acidez }{ }^{(* t}\right) \\
\text { (\% ac. oleico) }\end{array}$ & $\begin{array}{l}\text { Índice de peróxidos ("*) } \\
\left.\text { (meq } \mathrm{O}_{2} / \mathrm{kg}\right)\end{array}$ & $\begin{array}{l}\text { Absorción U.V. }\left({ }^{* *}\right) \\
\left(K_{270}\right)\end{array}$ \\
\hline 1 & $0.15 \pm 0.01^{a}$ & $6.6 \pm 0.5^{\mathrm{abc}}$ & $0.11 \pm 0.01^{\mathrm{ab}}$ \\
\hline 2 & $0.15 \pm 0.02^{a}$ & $7.0 \pm 0.4^{\mathrm{abc}}$ & $0.09 \pm 0.01^{a}$ \\
\hline 3 & $0.16 \pm 0.02^{a}$ & $5.7 \pm 0.3^{\mathrm{a}}$ & $0.18 \pm 0.06^{\mathrm{bcd}}$ \\
\hline 4 & $0.17 \pm 0.02^{\mathrm{ab}}$ & $6.0 \pm 0.7^{\mathrm{ab}}$ & $0.15 \pm 0.01^{\mathrm{abc}}$ \\
\hline 5 & $0.17 \pm 0.01^{\mathrm{ab}}$ & $7.3 \pm 0.4^{\mathrm{abc}}$ & $0.21 \pm 0.04^{\text {cde }}$ \\
\hline 6 & $0.16 \pm 0.01^{\mathrm{a}}$ & $7.2 \pm 0.5^{a b c}$ & $0.20 \pm 0.04^{\text {bode }}$ \\
\hline 7 & $0.18 \pm 0.02^{a b}$ & $6.9 \pm 0.7^{\mathrm{abc}}$ & $0.23 \pm 0.02^{\text {dde }}$ \\
\hline 8 & $0.17 \pm 0.02^{\mathrm{ab}}$ & $8.3 \pm 0.6^{c}$ & $0.24 \pm 0.02^{\text {dde }}$ \\
\hline 9 & $0.22 \pm 0.03^{b}$ & $6.9 \pm 1.1^{\mathrm{abc}}$ & $0.26 \pm 0.03^{d e}$ \\
\hline 10 & $0.19 \pm 0.01^{a b}$ & $7.3 \pm 0.6^{\mathrm{abc}}$ & $0.17 \pm 0.02^{a b c}$ \\
\hline 11 & $0.16 \pm 0.02^{\mathrm{a}}$ & $7.6 \pm 0.8^{\mathrm{bc}}$ & $0.18 \pm 0.04^{\mathrm{abod}}$ \\
\hline 12 & $0.17 \pm 0.02^{a}$ & $6.0 \pm 0.8^{a b}$ & $0.29 \pm 0.02^{e}$ \\
\hline 13 & $0.18 \pm 0.02^{a b}$ & $6.4 \pm 1.1^{\mathrm{abc}}$ & $0.28 \pm 0.04^{\ominus}$ \\
\hline Valor medio & $0.17 \pm 0.01$ & $6.9 \pm 0.2$ & $0.21 \pm 0.01$ \\
\hline $\begin{array}{l}\text { Semana de } \\
\text { recogida de } \\
\text { muestras }\end{array}$ & Grado de Acidez (*t) & İndice de peróxidos ("*) & $\left(K_{270}\right)\left({ }^{* *}\right)$ \\
\hline 1 & $0.15 \pm 0.00^{\mathrm{ab}}$ & $5.2 \pm 0.0^{\mathrm{a}}$ & $0.06 \pm 0.00^{a}$ \\
\hline 2 & $0.14 \pm 0.02^{a}$ & $7.7 \pm 0.7^{\mathrm{aod}}$ & $0.19 \pm 0.10^{\mathrm{a}}$ \\
\hline 3 & $0.15 \pm 0.01^{a b c}$ & $6.8 \pm 0.6^{\mathrm{ac}}$ & $0.26 \pm 0.06^{a}$ \\
\hline 4 & $0.15 \pm 0.01^{\mathrm{abc}}$ & $7.3 \pm 0.4^{\mathrm{ac}}$ & $0.20 \pm 0.02^{a}$ \\
\hline 5 & $0.16 \pm 0.01^{\mathrm{abc}}$ & $5.3 \pm 0.3^{\mathrm{ab}}$ & $0.23 \pm 0.03^{a}$ \\
\hline 6 & $0.17 \pm 0.02^{\mathrm{abcd}}$ & $6.2 \pm 0.3^{a b c}$ & $0.20 \pm 0.03^{\mathrm{a}}$ \\
\hline 7 & $0.16 \pm 0.01^{a b c}$ & $6.9 \pm 0.4^{\mathrm{ac}}$ & $0.22 \pm 0.03^{\mathrm{a}}$ \\
\hline 8 & $0.18 \pm 0.01^{\mathrm{abcd}}$ & $6.9 \pm 0.5^{\mathrm{ac}}$ & $0.20 \pm 0.04^{a}$ \\
\hline 9 & $0.21 \pm 0.02^{\mathrm{bde}}$ & $9.4 \pm 0.9^{d}$ & $0.21 \pm 0.03^{\mathrm{a}}$ \\
\hline 10 & $0.20 \pm 0.02^{\mathrm{abcde}}$ & $8.1 \pm 0.1^{\mathrm{a} a d}$ & $0.18 \pm 0.04^{\mathrm{a}}$ \\
\hline 11 & $0.25 \pm 0.06^{8}$ & $7.2 \pm 0.5^{\mathrm{ac}}$ & $0.16 \pm 0.06^{\mathrm{a}}$ \\
\hline Valor medio & $0.17 \pm 0.01$ & $6.9 \pm 0.2$ & $0.21 \pm 0.01$ \\
\hline
\end{tabular}

(*) Diferentes superíndices en una misma columna, indican diferencias significativas $(p<0.05)$ (**) Valor medio \pm desviación standard

En cuanto a la evolución de los índices de calidad a lo largo de la campaña (Tabla I), el análisis de la varianza ha mostrado diferencias significativas $(p<0.05)$ entre principio y final de la misma. Así el grado de acidez se sitúa en valores en torno a $0.15 \%$ al inicio de campaña, alcanzando el $0.25 \%$ al final de la misma (Semana 11). Este incremento del grado de acidez se puede atribuir a la propia evolución del fruto en el árbol, sometido a condiciones ambientales agresivas, propias de la recolección tardía, que favorecen la desestructuración de la epidermis de la oliva y, en consecuencia, la actividad lipolítica microbiana. Este incremento del grado de acidez conforme avanza la campaña, también se ha observado en los aceites de la zona del Montsià (De la Torre y López, 1986) y del Bajo Aragón (Ejarque, 1993). La evolución del índice de peróxidos y del $K_{270}$ a lo largo de la campaña, parece seguir una tendencia creciente, con ciertos incisos decrecientes, aunque las diferencias entre principio y final de campaña no son significativas $(p<0.05)$. La inestabilidad intrínseca de los peróxidos hace que el comportamiento de este índice, en función del tiempo de recolección, sea desigual, aún siguiendo una determinada línea evolutiva (Buron y García, 1979). El valor de $K_{270}$ relativamente elevado a principio de campaña (0.26 aproximadamente), podría estar relacionado con una recolección demasiado temprana, como la que se suele realizar en la D.O. para conseguir aceites más frutados. El descenso de este parámetro al final, 0.16 aproximadamente, podría ser consecuencia del menor contenido de pigmentos clorofílicos del aceite, que inducen a la fotooxidación en presencia de luz (Rahmani, 1989; Kiritsakis y Osman, 1995).

El contenido medio de polifenoles en la zona de la D.O. "Les Garrigues» para la campaña 1995/96 es de 152.4 ppm (Tabla II), concentración relativamente baja, teniendo en cuenta que el intervalo considerado como normal para el aceite de oliva virgen está entre 50 y 500 ppm (Vázquez et al., 1973). Tal y como se observa en la Tabla II, el rango de concentración en las almazaras muestreadas es muy amplio, observándose valores entre 82.4 ppm y 304,6 ppm, lo que ha motivado que el análisis de la varianza para este parámetro se haya realizado con un nivel de significación del $99 \%$. Esta variabilidad no se ha podido relacionar con la localización de la almazara, hecho lógico si tenemos en cuenta que la Denominación de Origen, como tal, comprende una zona geográfica limitada con características geoclimáticas y de cultivo del olivo similares. En este sentido, se ha observado que la variedad de la oliva de origen así como la zona de cultivo son factores determinantes del contenido de polifenoles de un aceite (Solinas, 1987). Sin embargo, en nuestro estudio la tecnología de extracción del aceite parece tener una gran influencia. Así, los aceites obtenidos mediante el sistema de extracción continua de dos fases tienen un contenido medio de polifenoles de $174.3 \mathrm{ppm}$, cantidad significativamente superior $(p<0.01)$ que los obtenidos por el sistema de tres fases, con un valor medio de $128.4 \mathrm{ppm}$. La mayor cantidad de agua que se adiciona a la pasta en este último sistema, favorece que los compuestos polifenólicos de naturaleza hidrosoluble pasen a la fase acuosa y se pierdan con los alpechines (Hermoso et al., 1995, Di Giovacchino, 1996). No obstante, algunas almazaras de dos fases, como la 9 ( 99.6 ppm) tienen un contenido de polifenoles inferior a algunas de tres fases, probablemente relacionado con una mayor adición de agua o bien con otros parámetros tecnológicos (temperatura y tiempo de batido, etc.) que pueden influir en la cantidad de estos componentes de la oliva que pasan al aceite durante el proceso de extracción (Montedoro, 1973, Solinas et al., 1975 a). 
Tabla II

Contenido de polifenoles, expresado en $\mathrm{mg}$ de ácido cafeico/kg de aceite (ppm), del aceite de oliva virgen del período de recogida de muestras de las almazaras estudiadas en la D.O. “Les Garrigues», durante la campaña 1995/96 (*)

\begin{tabular}{|c|c|c|}
\hline Almazara & $\begin{array}{l}\text { Sistema de extracción (") } \\
\text { (Fases) }\end{array}$ & $\begin{array}{l}\text { Polifenoles ("*) } \\
\text { (ppm) }\end{array}$ \\
\hline 2 & 2 & $166.2 \pm 7.5^{\mathrm{a}}$ \\
\hline 3 & 2 & $304.6 \pm 9.9^{b}$ \\
\hline 4 & 2 & $170.8 \pm 11.0^{\mathrm{a}}$ \\
\hline 5 & 2 & $126.0 \pm 4.2^{\mathrm{co}}$ \\
\hline 6 & 2 & $170.4 \pm 8.6^{\mathrm{a}}$ \\
\hline 9 & 2 & $99.6 \pm 12.3^{\text {de }}$ \\
\hline 13 & 2 & $149.2 \pm 12.0^{\text {ac }}$ \\
\hline 1 & 3 & $222.1 \pm 7.0^{\prime}$ \\
\hline 7 & 3 & $82.4 \pm 10.8^{d}$ \\
\hline 8 & 3 & $115.6 \pm 8.9^{\mathrm{ce}}$ \\
\hline 10 & 3 & $99.3 \pm 10.2^{\text {de }}$ \\
\hline 11 & 3 & $145.0 \pm 7.5^{\mathrm{ac}}$ \\
\hline 12 & 3 & $112.1 \pm 4.6^{\mathrm{cde}}$ \\
\hline Valor medio & & $152.4 \pm 2.6$ \\
\hline \multicolumn{2}{|c|}{ Semana de recogida de muestras } & $\begin{array}{l}\text { Polifenoles ("*) } \\
\text { (ppm) }\end{array}$ \\
\hline \multicolumn{2}{|r|}{1} & $320.9 \pm 24.2^{\mathrm{a}}$ \\
\hline \multicolumn{2}{|r|}{2} & $199.2 \pm 33.7^{\mathrm{abd}}$ \\
\hline \multicolumn{2}{|r|}{3} & $155.4 \pm 19.3^{\text {bode }}$ \\
\hline \multicolumn{2}{|r|}{4} & $150.4 \pm 10.3^{\text {bode }}$ \\
\hline \multicolumn{2}{|r|}{5} & $181.4 \pm 14.9^{\text {bd }}$ \\
\hline \multicolumn{2}{|r|}{6} & $164.3 \pm 22.7^{\text {bode }}$ \\
\hline \multicolumn{2}{|r|}{7} & $120.9 \pm 12.9^{\text {cdo }}$ \\
\hline \multicolumn{2}{|r|}{8} & $138.6 \pm 12.3^{\text {bode }}$ \\
\hline \multicolumn{2}{|r|}{9} & $99.6 \pm 11.5^{\circ}$ \\
\hline \multicolumn{2}{|c|}{10} & $133.5 \pm 2.2^{\text {bede }}$ \\
\hline \multicolumn{2}{|c|}{11} & $143.2 \pm 35.9^{\text {bode }}$ \\
\hline \multicolumn{2}{|c|}{ Valor medio } & $152.4 \pm 2.6$ \\
\hline
\end{tabular}

(*) Diferentes superindices en una misma columna, indican diferencias significativas $(p<0.01)$ (**) Valor medio \pm desviación standard

El contenido de polifenoles del aceite a lo largo de la campaña se mantiene más o menos estable, con una cierta tendencia a disminuir (Tabla II). Sin embargo, el análisis de la varianza no ha presentado diferencias significativas $(p<0.01)$ entre ambas variables, a excepción de la semana 1 en que el contenido medio es de 320.9 ppm y la semana 9 con 99.6 $\mathrm{ppm}$. Esta tendencia, aunque no significativa estadísticamente, puede estar relacionada con el momento de la recolección de la oliva que se inicia en la denominada madurez crítica, cuando su contenido en aceite es máximo. La concentración de polifenoles aumenta a lo largo de la pigmentación, y también es máxima en este momento (Solinas et al., 1975 b; Chimi y Atouati, 1994; Maestro-Durán et al., 1994).

La concentración media de clorofilas y carotenoides en el aceite para esta campaña se sitúa en $9.9 \mathrm{mg} / \mathrm{kg}$ y $7.5 \mathrm{mg} / \mathrm{kg}$ de aceite, respectivamente
(Tabla III). No se observan grandes diferencias entre las cooperativas muestreadas, a excepción de la almazara 3 que tiene la concentración más elevada de pigmentos $(11.9 \mathrm{mg}$ clorofilas $/ \mathrm{kg}$ y $9.3 \mathrm{mg}$ carotenoides $/ \mathrm{kg}$ ) y, en el extremo opuesto, la almazara 12 que presenta las concentraciones más bajas $(6.8 \mathrm{mg}$ clorofilas $/ \mathrm{kg}$ y $5.4 \mathrm{mg}$ carotenoides $/ \mathrm{kg}$ ). Estas diferencias no se han podido explicar en función de factores geográficos, climáticos y tecnológicos. En cuanto a la evolución del contenido de pigmentos en función de la época de campaña, se observa una tendencia decreciente, especialmente marcada a partir de las semanas 5 y 6 de recolección. El rango de variación presenta un máximo de $15.8 \mathrm{mg}$ clorofilas $/ \mathrm{kg}$ y 12.6 $\mathrm{mg}$ carotenoides $/ \mathrm{kg}$, que desciende hasta un mínimo de $5.9 \mathrm{mg}$ clorofilas $/ \mathrm{kg}$ y $4.6 \mathrm{mg}$ carotenoides $/ \mathrm{kg}$, al final de la campaña (Tabla III). La tendencia en la disminución de pigmentos en el aceite de oliva parece seguir un comportamineto similar al observado por Minguez et al. (1991) para la variedad «Hojiblanca», aunque con un rango de variación mucho más amplio. El descenso en la concentración de clorofilas se podría explicar por la actuación de varias enzimas de degradación presentes en la oliva. La pérdida de pigmentos clorofílicos conforme avanza la maduración, provoca la atenuación del color verde en la oliva y, en consecuencia la variación del color del aceite en función de las cantidades y proporciones de otros pigmentos, principalmente compuestos de degradación (Mínguez et al., 1990).

La distribución porcentual en el aceite de pigmentos clorofílicos y carotenoides a lo largo de la campaña es aproximadamente del $57 \%$ y del $43 \%$, respectivamente, y se mantiene prácticamente constante (Tabla III). Esta estabilidad no coincide con la descrita por Mínguez et al. (1991) que observan una mayor reducción en la proporción de la fracción clorofílica en el aceite, en fechas tardías de recolección de la oliva. A la vista de los resultados, es posible deducir que en la variedad "Arbequina» los pigmentos, tanto clorofilas como carotenoides, permanecen en compartimentos individuales equilibrados en el interior de los cloroplastos, de manera que aunque su concentración disminuye conforme avanza la campaña, su proporción relativa se mantiene prácticamente fija. Por tanto, el cambio de tonalidad que se observa entre aceites de principio y final de campaña, podría ser consecuencia del incremento porcentual de alguno de los pigmentos derivados que integran las fracciones clorofílicas y carotenoides, y que tienen diferentes propiedades espectrales.

Los resultados del estudio del color del aceite estan basados en la evaluación subjetiva, mediante el índice ABT, y en las coordenadas instrumentales CIELAB (Tabla IV). El valor que aparece con mayor frecuencia como media del índice ABT en las cooperativas muestredas, es el 2/6-2/7, típico de la variedad «Arbequina» (Tous y Romero, 1992). No obstante, 
destacan las medias de las almazaras 4 y 6 , situadas en la serie tercera, con tonos más verdes que el resto. En cuanto a la evolución de este índice a lo largo de la campaña, no se aprecian cambios importantes en la tonalidad de los aceites (numerador), tan sólo se observa una ligera disminución de la luminosidad (denominador), a diferencia de algunas campañas precedentes (1992/93) en las que los aceites de principio han presentado colores más verdosos que los del final, más amarillos (Solé, 1995). El hecho de que el índice ABT, en este caso, no distinga entre el aceite de principio de campaña (tonos verdosos y menor claridad) y el de final (amarillo y más claro), se puede atribuir a la climatología anómala de esta campaña que ha propiciado un avanzado estado de madurez de las olivas en el inicio de la recolección.

Tabla III

Contenido en pigmentos clorofílicos y carotenoides, expresado en $\mathrm{mg} / \mathrm{kg}$, del aceite de oliva virgen del período de recogida de muestras de las almazaras estudiadas en la D.O. «Les Garrigues» durante la campaña 1995/96 (*)

\begin{tabular}{|c|c|c|c|c|}
\hline \multirow{2}{*}{ Almazara } & \multirow{2}{*}{$\begin{array}{c}\text { Clorofilas ("t) } \\
\text { (mg/kg) }\end{array}$} & \multirow{2}{*}{$\begin{array}{l}\text { Carotenoides }(* *) \\
(\mathrm{mg} / \mathrm{kg})\end{array}$} & \multicolumn{2}{|c|}{ Pigmentos totales } \\
\hline & & & $\%$ clorofilas & $\%$ carotenos \\
\hline 1 & $9.0 \pm 1.2^{\mathrm{ab}}$ & $7.9 \pm 0.5^{\mathrm{bcd}}$ & 53 & 47 \\
\hline 2 & $8.3 \pm 0.7^{\mathrm{ab}}$ & $6.9 \pm 0.5^{\mathrm{abc}}$ & 55 & 45 \\
\hline 3 & $11.9 \pm 1.4^{b}$ & $9.3 \pm 0.9^{d}$ & 56 & 44 \\
\hline 4 & $10.3 \pm 2.1^{a b}$ & $8.3 \pm 1.3^{\text {bcd }}$ & 55 & 45 \\
\hline 5 & $8.8 \pm 0.6^{\mathrm{ab}}$ & $6.8 \pm 0.5^{\mathrm{abc}}$ & 56 & 44 \\
\hline 6 & $11.8 \pm 1.7^{b}$ & $8.5 \pm 1.0^{\text {bed }}$ & 58 & 42 \\
\hline 7 & $10.1 \pm 1.1^{\mathrm{ab}}$ & $6.9 \pm 0.7^{\mathrm{abc}}$ & 59 & 41 \\
\hline 8 & $10.3 \pm 1.3^{a b}$ & $6.9 \pm 0.8^{a b c}$ & 60 & 40 \\
\hline 9 & $9.6 \pm 1.2^{a b}$ & $6.4 \pm 0.9^{\mathrm{ab}}$ & 60 & 40 \\
\hline 10 & $9.2 \pm 0.7^{a b}$ & $7.6 \pm 0.5^{\mathrm{abod}}$ & 55 & 45 \\
\hline 11 & $11.6 \pm 1.3^{b}$ & $8.8 \pm 0.7^{\text {od }}$ & 57 & 43 \\
\hline 12 & $6.8 \pm 0.5^{\mathrm{a}}$ & $5.4 \pm 0.2^{\mathrm{a}}$ & 56 & 44 \\
\hline 13 & $10.9 \pm 2.0$ ab & $8.1 \pm 1.0^{\mathrm{abad}}$ & 57 & 43 \\
\hline Valor medio & $9.9 \pm 0.4$ & $7.5 \pm 0.2$ & 57 & 43 \\
\hline \multirow{2}{*}{$\begin{array}{l}\text { Semana de } \\
\text { recogida de } \\
\text { muestras }\end{array}$} & \multirow{2}{*}{$\begin{array}{c}\text { Clorofilas ("*) } \\
(\mathrm{mg} / \mathrm{kg})\end{array}$} & \multirow{2}{*}{$\begin{array}{c}\text { Carotenoides }(* *) \\
(\mathrm{mg} / \mathrm{kg})\end{array}$} & \multicolumn{2}{|c|}{ Pigmentos totales } \\
\hline & & & $\%$ clorofilas & $\%$ carotenos \\
\hline 1 & $15.8 \pm 0.0^{\circ}$ & $12.6 \pm 0.0^{\circ}$ & 56 & 44 \\
\hline 2 & $13.0 \pm 0.6^{\mathrm{de}}$ & $9.9 \pm 0.5^{\mathrm{de}}$ & 57 & 43 \\
\hline 3 & $11.7 \pm 1.2^{\mathrm{de}}$ & $8.7 \pm 0.6^{d}$ & 57 & 43 \\
\hline 4 & $11.6 \pm 0.7^{d e}$ & $8.5 \pm 0.4^{d}$ & 58 & 42 \\
\hline 5 & $10.2 \pm 1.2^{\mathrm{cd}}$ & $8.4 \pm 0.6^{d}$ & 55 & 45 \\
\hline 6 & $9.5 \pm 0.4^{\mathrm{bcd}}$ & $6.7 \pm 0.4^{c}$ & 59 & 41 \\
\hline 7 & $8.8 \pm 0.7^{a b c}$ & $6.5 \pm 0.3^{b c}$ & 58 & 42 \\
\hline 8 & $8.0 \pm 0.5^{\mathrm{abc}}$ & $6.3 \pm 0.3^{a b c}$ & 56 & 44 \\
\hline 9 & $7.0 \pm 0.7^{\mathrm{ab}}$ & $5.1 \pm 0.3^{\mathrm{ab}}$ & 58 & 42 \\
\hline 10 & $7.5 \pm 0.3^{\mathrm{abc}}$ & $5.8 \pm 0.1^{\mathrm{abc}}$ & 56 & 44 \\
\hline 11 & $5.9 \pm 0.1^{\mathrm{a}}$ & $4.6 \pm 0.6^{\mathrm{a}}$ & 56 & 44 \\
\hline Valor medio & $9.9 \pm 0.3$ & $7.5 \pm 0.2$ & 57 & 43 \\
\hline
\end{tabular}

(*) Diferentes superindices en una misma columna, indican diferencias significativas $(p<0.05)$ (**) Valor medio \pm desviación standard
Tabla IV

Color, expresado según la escala ABT y las ordenadas cromáticas $a^{\star}, b^{\star}, L^{*}$ y $C^{\star}$ del aceite de oliva virgen del período de recogida de muestras de las almazaras estudiadas en la D.O. «Les Garrigues», durante la campaña 1995/96 (*)

\begin{tabular}{|c|c|c|c|c|c|}
\hline \multirow{2}{*}{ Almazara } & \multirow{2}{*}{ ABT } & \multicolumn{4}{|c|}{ Ordenadas cromáticas } \\
\hline & & $a^{*}$ & $b^{*}$ & $\mathrm{~L}^{*}$ & $\mathrm{C}^{*}$ \\
\hline 1 & $2 / 6$ & $-1.38^{\text {abod }}$ & $98.2^{d e}$ & $84.7^{\mathrm{ab}}$ & $98.2^{d \theta}$ \\
\hline 2 & $2 / 6$ & $-2.32^{\mathrm{abc}}$ & $89.0^{\mathrm{abadt}}$ & $87.4^{a b}$ & $88.9^{a b c d}$ \\
\hline 3 & $2 / 7$ & $-0.77^{\mathrm{abcd}}$ & $102.7^{\ominus}$ & $86.6^{\mathrm{ab}}$ & $102.8^{\mathrm{e}}$ \\
\hline 4 & $3 / 6$ & $-0.11^{\text {od }}$ & $81.2^{\mathrm{ab}}$ & $77.8^{\mathrm{a}}$ & $81.3^{a b}$ \\
\hline 5 & $2 / 9$ & $-4.05^{\mathrm{a}}$ & $82.8^{a b}$ & $86.2^{\mathrm{ab}}$ & $83.0^{\mathrm{ab}}$ \\
\hline 6 & $3 / 2$ & $-0.53^{\text {bod }}$ & $91.2^{\text {bed }}$ & $80.2^{a b}$ & $91.2^{\text {bed }}$ \\
\hline 7 & $2 / 7$ & $-2.51^{a b c}$ & $82.7^{\mathrm{ab}}$ & $86.8^{\mathrm{ab}}$ & $82.7^{\mathrm{ab}}$ \\
\hline 8 & $2 / 6$ & $-2.22^{a b c}$ & $78.7^{\mathrm{a}}$ & $86.1^{\mathrm{ab}}$ & $78.7^{\mathrm{a}}$ \\
\hline 9 & $2 / 6$ & $-3.40^{\mathrm{ab}}$ & $79.0^{\mathrm{a}}$ & $86.8^{\mathrm{ab}}$ & $79.1^{\mathrm{a}}$ \\
\hline 10 & $2 / 6$ & $-0.66^{\mathrm{abcd}}$ & $89.1^{\text {abod }}$ & $83.2^{a b}$ & $89.2^{a b c d}$ \\
\hline 11 & $2 \pi$ & $1.42^{d}$ & $86.1^{\mathrm{abc}}$ & $76.0^{\mathrm{a}}$ & $86.2^{a b c}$ \\
\hline 12 & $2 / 6$ & $-3.52^{a b}$ & $79.5^{\mathrm{a}}$ & $90.8^{b}$ & $79.6^{\mathrm{a}}$ \\
\hline 13 & $2 / 7$ & $-1.39^{\mathrm{abcd}}$ & $98.6^{\text {de }}$ & $89.4^{a b}$ & $98.6^{\text {cde }}$ \\
\hline Valor medio & $2 / 6$ & -1.63 & 87.0 & 84.5 & 87.0 \\
\hline \multirow{3}{*}{ Semana } & & \multicolumn{4}{|c|}{ Ordenadas cromáticas } \\
\hline & ABT & & & & \\
\hline & & $a^{*}$ & $\mathbf{b}^{*}$ & $L^{*}$ & $\mathrm{C}^{*}$ \\
\hline 1 & $2 / 8$ & $3.78^{b}$ & $101.8^{\text {d }}$ & $73.3^{a}$ & $101.9^{\mathrm{dd}}$ \\
\hline 2 & $2 \pi$ & $1.03^{b}$ & $96.3^{\text {d }}$ & $79.7^{\mathrm{abc}}$ & $96.3^{\text {dd }}$ \\
\hline 3 & $2 / 6$ & $-0.29^{b}$ & $97.6^{d}$ & $81.3^{\mathrm{abcd}}$ & $97.7^{d}$ \\
\hline 4 & $2 / 6$ & $0.47^{b}$ & $88.7^{b c}$ & $78.2^{\mathrm{ab}}$ & $88.7^{b c}$ \\
\hline 5 & $2 \pi$ & $-0.60^{b}$ & $86.9^{b c}$ & $77.2^{\mathrm{ab}}$ & $86.9^{\mathrm{bc}}$ \\
\hline 6 & $2 / 6$ & $-2.98^{a}$ & $86.4^{\mathrm{bc}}$ & $88.2^{\text {acde }}$ & $86.3^{\text {bc }}$ \\
\hline 7 & $2 \pi 7$ & $-2.98^{a}$ & $89.6^{\circ}$ & $90.4^{c e}$ & $89.6^{c}$ \\
\hline 8 & $2 / 7$ & $-3.46^{a}$ & $87.1^{b c}$ & $91.1^{c \theta}$ & $87.1^{b c}$ \\
\hline 9 & $2 / 5$ & $-4.08^{a}$ & $68.8^{a}$ & $92.8^{\circ}$ & $68.9^{\mathrm{a}}$ \\
\hline 10 & $2 / 5$ & $-4.52^{\mathrm{a}}$ & $76.8^{a b}$ & $92.3^{\text {cde }}$ & $77.0^{\mathrm{ab}}$ \\
\hline 11 & $2 / 5$ & $-4.81^{a}$ & $66.4^{\mathrm{a}}$ & $94.0^{\circ}$ & $66.6^{\mathrm{a}}$ \\
\hline Valor medio & - & -1.63 & 87.0 & 84.5 & 87.1 \\
\hline
\end{tabular}

(*) Diferentes superindices en una misma columna, indican diferencias significativas $(p<0.05)$

El color, en base a las coordenadas CIELAB, no presenta grandes diferencias entre almazaras (Tabla IV). El parámetro $\mathrm{a}^{*}$ tiene un valor medio de -1.63 , que supera ligeramente el eje de los tonos verdes; el análisis de la varianza no ha mostrado diferencias significativas $(p<0.05)$, a excepción de la almazara 5 , con un valor medio de $a^{*}$ de -4.05 situado en la zona de tonos verdes y la almazara 11, con un valor de $a^{*}$ de 1.42 situado en la zona de tonos rojos. En relación con la coordenada $\mathrm{b}^{\star}$, todas las medias están situadas en la zona de amarillos, con un valor medio para esta campaña de 87.0, destacando la almazara 3 con un valor de 102.7 para este parámetro. Curiosamente, se observa una cierta tendencia de $a^{*}$ hacia valores negativos (zona verde) y de $b^{*}$ hacia la zona del azul, en los aceites con contenidos más bajos de pigmentos clorofílicos y carotenoides (almazaras 5 y 12). El valor medio de la coordenada $b^{*}$ 
es similar al observado por Mínguez et al. (1991) para la variedad Hojiblanca recogida en el mes de diciembre y muy superior al correspondiente a otras variedades de oliva andaluzas (Lechín, Verdial y Picudo). Sin embargo, los valores de la coordenada $a^{*}$ son similares a los observados en la variedad Hojiblanca en un período de recolección más tardío (febrero). La coordenada $L^{*}$ no muestra diferencias dignas de reseñar entre almazaras, las medias se mantienen dentro de un rango de valores delimitado y no muy amplio, comprendido entre 76 y 90 . El parámetro $C^{*}$ presenta unas medias casi idénticas a las de la coordenada $b^{*}$; el análisis de la varianza no ha mostrado grandes diferencias, tan sólo la almazara 3 tiene una valor medio de croma de 102.8, ligeramente superior al resto.

El estudio de la evolución del color del aceite a lo largo de la campaña, en base a las coordenas CIE$L A B$, ha mostrado diferencias significativas $(p)$ entre principio y final de la misma (Tabla IV). La variable $a^{*}$ se desplaza desde valores positivos situados en los tonos rojos (3.78 en la semana 1) hasta tonos ligeramente verdes (-4.81 en la semana 11). Sin embargo, el rango de variación es tan pequeño que difícilmente puede tener implicaciones en la apreciación del color del aceite, y el hecho de que los valores se mantengan en todo momento próximos a la zona del rojo en el transcurso de la campaña, puede ser consecuencia de la avanzada maduración de la oliva al inicio de la misma. Los valores de $b^{*}$ se reducen significativamente a lo largo de la campaña (101.8 en la semana 1 a 66.4 en la semana 11), evolucionando hacia el azul, aunque siempre se mantiene en valores positivos dentro de la zona del amarillo. Paralelamente, la coordenada $C^{*}$, que expresa la saturación o pureza del color, sigue una pauta descendente casi idéntica a la de $b^{\star}$, evolucionando hacia colores más grises y menos puros. La evolución de estos parámetros, aunque no detectable por simple observación visual en la escala $A B T$, podría ser consecuencia de la presencia de compuestos de degradación de los pigmentos clorofílicos y carotenoides, en estados más avanzados de maduración de la oliva al final de la campaña.

A la vista de los resultados, el estudio muestra que los aceites de oliva de la D.O. "Les Garrigues» de la campaña 1995/96 tienen un grado de acidez medio de $0.17 \%$, índice de peróxidos de $6.9 \mathrm{meq} / \mathrm{kg}$ de aceite y un valor medio de K270 de 0.21 , valores todos ellos dentro de los criterios de calidad que establece la Reglamentación de la Denominación de Origen (B.O.E, 13 de julio de 1977). El contenido medio de polifenoles para esta campaña se sitúa en torno a $152.4 \mathrm{ppm}$, con valores que oscilan entre 82.4 y 304.6 ppm. Esta variabilidad entre cooperativas, probablemente es consecuencia de las condiciones de proceso en la extracción del aceite (sistema de centrifugación, temperatura de batido, cantidad de agua añadida, etc.). La concentración de pigmentos clorofílicos y carotenoides del aceite desciende conforme avanza la campaña, no presentando grandes diferencias entre las diferentes zonas de la D.O. La concentración de clorofilas se reduce desde valores en torno a $15 \mathrm{mg} / \mathrm{kg}$ de aceite, al inicio de campaña hasta $6 \mathrm{mg} / \mathrm{kg}$ al final de la misma. La concentración de carotenoides se reduce paralelamente, pasando de $12.6 \mathrm{mg} / \mathrm{kg}$ a $4.6 \mathrm{mg} / \mathrm{kg}$ al final de campaña. No se observan grandes diferencias de color entre aceites de principio y final de campaña, probablemente como consecuencia del avanzado estado de maduración al inicio de campaña que ha caracterizado este año.

\section{AGRADECIMIENTOS}

Los autores agradecen al Consejo Regulador de la Denominación de Origen «Les Garrigues» la recogida de muestras de aceite y a las cooperativas participantes en este estudio su colaboración técnica. Al Centro de Referencia en Tecnología de Alimentos (CeRTA) de la Generalitat de Cataluña por el soporte económico que ha permitido la realización del trabajo.

\section{BIBLIOGRAFÍA}

Angerosa, F. y Di Giovacchino, L. (1996).—“Natural antioxidants of virgin olive oil obtained by two and tri phase centrifugal decanters".-Grasas y Aceites 47 247-254.

Beltrán, G., Jiménez, A. y Uceda, M. (1995).——Efecto del régimen hídrico de cultivo sobre la fracción fenólica del aceite de oliva de la variedad arbequina".- I Simposi de l'olivera a Catalunya. pp. 153-155. Les Borjes Blanques, Lleida.

BOLETIN OFICIAL DEL ESTADO (B.O.E.). ORDEN 10 de mayo de 1977. ACEITE Reglamento de la Denominación de origen «Borjas Blancas» y de su Consejo Regulador.

Buron, I. y García, R. (1979).— «La calidad del aceite de oliva".-Ed. Instituto Nacional de Investigaciones Agrarias. Madrid.

Chimi, H. y Atouati, B.Y. (1994).-—Determinación de la fase óptima de recogida de las aceitunas de la variedad Picholine marroquí mediante el seguimiento de la evolución de los polifenoles totales".-Olivae 54, 56-60.

De La Torre, M.C. y López, C. (1986).-—Estudi de la qualitat dels olis de Catalunya".-Sessions tecniques de l'olivera. Ed. Obra Agrícola de "La Caixa».

DIARIO OFICIAL DE LAS COMUNIDADES EUROPEAS (D.O.C.E.). REGLAMENTO (CEE) N. 2568/91 DE LA COMISION de 11 de julio de 1991 relativo a las características de los aceites de oliva y de los aceites de orujo de oliva y sobre sus métodos de análisis. Anexos II, III y IX. 
Di Giovacchino, L. (1991).- «Extracción del aceite de las aceitunas por presión, centrifugación y percolación; efectos de las técnicas sobre los rendimientos en aceite".-Olivae 36, 42-44.

Di Giovacchino, L. (1996).- - Influencia de los sistemas de extracción en la calidad del aceite de oliva».-Olivae $63,52-56$.

Ejarque, C. (1993).—-Calidad fisico-química del aceite de oliva virgen del Bajo Aragón turolense».-Trabajo Final de Carrera. Escuela Técnica Superior de Ingeniería Agraria. Universidad de Lleida.

García Guillamet, G. (1995).— «Situación actual y perspectivas de futuro del sector olivarero en las Denominaciones de origen: Garrigues y Siurana».-I Simposi de l'olivera Arbequina en Cataluña. Les Borges Blanques, Lleida.

Graell, J. (1992)._- «Calidad del aceite de oliva virgen D.O. Borjas Blancas. Influencia del proceso de extracción".-II Forum Oleícola Internacional. II Salón Internacional Oleícola (SIO). Reus (Tarragona).

Graell, J., Arbos, J., Duro, V., Clota, J., Torrelles, R., Ciria, R., Puig, J. y Munté, M. (1993 a).—-Calidad del aceite de oliva virgen D.O. Borjas Blancas. I. Medio físico y cultivo. Proceso de elaboración».-Alimentación, equipos y tecnología 9, 97-103.

Graell, J., Arbos, J., Duro, V., Clota, J., Torrelles, R., Ciria, R., Puig, J. y Munté, M. (1993 b).— «Calidad del aceite de oliva virgen D.O. Borjas Blancas. III. Influencia del proceso de extracción».-Alimentación, equipos y tecnología 10, 119-127.

Gutiérrez, R y Gutiérrez, F. (1986).— «Método rápido para definir y clasificar el color de los aceites de oliva vírgenes".-Grasas y Aceites 37, 282-284.

Hermoso, M., Uceda, M., González, J. y Morales, J. (1995).— «Industria de elaboración de aceite de oliva".-Agricultura. Suplemento de mayo, 81-83.

Kiritsakis, A. y Osman, M. (1995).- - Efectos del $\beta$-caroteno y del $\alpha$-tocoferol en la estabilidad fotooxidativa del aceite de oliva".-Olivae, 56, 25-28.

Maestro-Durán, R., León Cabello, R. y Ruíz Gutierrez, V. (1994)._- «Compuestos fenólicos del olivo (Olea europaea)".-Grasas y Aceites 45, 265-269.

Mínguez, M.I., Rejano, L., Gandul, B., Sánchez, A. y Garrido, J. (1990).— «Pigments present in virgin olive oil».-J. Am. Oil Chem. 67, 192-196.

Mínguez, M.I., Rejano, L., Gandul, B., Sánchez, A. y Garrido, J. (1991).- "Color-Pigment correlation in virgin olive oil».-J. Am. Oil Chem. 68, 332-336.

Montedoro, G. (1973).- «Esame analitico dei costituenti fenolici presenti nell olio di oliva in funzione delle caratteristiche della drupa, loro ruolo nella stabilita all ossidazione e possibile interferenza nella determinazione del numero di perossidi».-Estratto dagli Annali della Facolta di Agraria dell Universita di Perugia, Vol XXVIII, 1-20.

Papaseit, J. (1986).— «El color del aceite de oliva extra virgen, característica de calidad".-Grasas y Aceites, 37, 204-206.
Pujades, R. (1984).— «Les Garrigues: Diagnosi comarcal».Ed. Departament de Política Territorial y Obres Publiques de la Generalitat de Cataluña.

Rahmani, M. (1989)._ «Puntualización sobre la función de los pigmentos clorofílicos en la fotooxidación del aceite de oliva virgen".-Olivae, 26, 30-33.

Romero, A., Tous, J., Plana, J. y Díaz, I. (1995 a)."Caracterización físico-química de los aceites de la variedad "Arbequina"»._- Simposi de l'olivera Arbequina en Cataluña. pp. 127-129. Les Borges Blanques, Lleida.

Romero, M.P., Motilva, M.J. y Graell, J. (1995 b)."Calidad del aceite de oliva virgen (variedad Arbequina) elaborado en distintas almazaras de D.O. Garrigues".- - Simposi de l'olivera Arbequina en Cataluña. pp. 159-162. Les Borges Blanques, Lleida.

Solé, M.A. (1995 a).- «Caracterización organoléptica de los aceites de oliva virgen extra de la variedad "Arbequina" de la zona de "Les Garrigues".Simposi de l'olivera Arbequina en Cataluña. pp. 149-152. Les Borges Blanques, Lleida.

Solé, M.A. (1995 b).- - Algunas observaciones sobre la evolución del color del aceite de la variedad "Arbequina» en la comarca de Les Garrigues durante el período de recolección".- - Simposi de l'olivera Arbequina en Cataluña. pp. 163-166. Les Borges Blanques, Lleida.

Solinas, M., Di Giovacchino, L. y Cucurachi, A. (1975 a).——I Polifenoli delle olive e dell olio di oliva. Nota $2{ }^{\mathrm{a}}$ : Indagine preliminare sull incidenza delle tecnique operative adottate per l'estrazione dell olio".-Estratto dagli Annali dell Istituto Sperimentale per la Elaiotecnica-Pescara, Pubblicazione N. 19, Vol V, 1-26.

Solinas, M., Di Giovacchino, L. y Cucurachi, A. (1975 b).- «l Polifenoli delle olive e dell olio di oliva. Nota 1․: Variazioni che subiscono alcuni polifenoli delle olive col procedere della maturazione».-Estratto dagli Annali dell Istituto Sperimentale per la ElaiotecnicaPescara, Pubblicazione N. 18, Vol V, 1-24.

Solinas, M. (1987).-«Analisi HRGC delle sostanze fenoliche di oli vergini di oliva in relazione al grado di maturazione e alla varieta delle olive».-La Rivista delle Sostanze Grasse, 64, 255-262.

Tous, J. y Romero, A. (1992).- «Caracterización del color de los aceites de oliva vírgenes de cultivares catalanes".-Grasas y Aceites, 43, 347-351.

Tous, J. y Romero, A. (1993).- - «Variedades del olivo. Con especial referencia a Cataluña».-Ed. Fundació «La Caixa»-AEDOS. Barcelona, $172 \mathrm{p}$.

Tous, J. y Romero, A. (1994).- "Cultivar and location effects on olive oil quality in Catalonia (Spain)».-Acta Horticulturae, 356, 323-326.

Vázquez, A., Janer del Valle, C., Janer del Valle, L. (1973).- «Determinación de los polifenoles totales en aceite de oliva".-Grasas y Aceites, 24, 350-357.

Recibido: Octubre 1997 Aceptado: Febrero 1998 